\title{
Breve recorrido histórico-lingüístico en la Sicilia de la Guerra de las Vísperas
}

\author{
Salvatore BartolotTa \\ UNED
}

\begin{abstract}
RESUMEN ABSTRACT
Desde el punto de vista de la historia lingüistica, 1282 representa un cambio significativo en la vida siciliana. Además de una dirección politica diferente, y por lo tanto lingüistica (hacia la Península Ibérica más que hacia Francia), que la isla empezó a experimentar y que probablemente permaneceria durante algunos siglos, la consecuencia más significativa de las Vísperas, por lo que nos interesa, fue la ruptura de relaciones con la parte meridional de la península.

\section{PALABRAS CLAVE}

angevinos, Anjou, árabe, aragoneses, Aviñón, Caltabellotta, dialecto, griego,

guerra, latín, lengua escrita, lengua hablada, lírica, neolatino, normanda, policentrismo, prestigio, prosa, Sicilia, siciliano, sueva, Visperas.

From the point of view of linguistic history, 1282 represents a significant change in Sicilian life. Apart form a different political direction, and therefore linguistic (towards the Iberian Peninsula more than towards France), that the island started to experiment and that would probably remain for a few centuries, the most significant consequence of the Vespers, as far as we are concerned, was the break-up of relations with the southern part of the peninsula.

\section{KEYWORDS}

Angevine, Anjou, Arab, Aragonese, Avignon, Caltabellotta, dialect, Greek, war, Latin, written language, spoken language, poetry, Neolatin, Norman, policentrism, prestige, prose, Sicily, Sicilian, Swabian, Vespers.
\end{abstract}

"Se, come per altre realtà regionali, il policentrismo costituisce l'aspetto principale della storia politica e culturale della Sicilia - che è, ancora più che altrove, storia tumultuosa di invasioni e conquiste da parte di popolazioni assai dissimili per costumi, religione e lingua - , l'insularità del territorio fisico, contrariamente a quanto è stato anche autorevolmente sostenuto e forse si continua a pensare, non è tratto pertinente della sua cultura, che pure mostra una precisa e talora risentita autonoma identità in perenne tensione con quella dell'intera nazione. 
In effetti, anche sotto il rispetto linguistico, questo presunto isolamento non trova nessuna rispondenza nella realtà, essendo privo di qualsiasi fondamento scientifico di confine dialettale convenzionalmente rappresentato dallo stretto di Messina (e dai limiti dell'isola), data la notevole congruenza fenomenica tra le parlate delle due sponde (e delle isole minori) »'.

MATTESINI (1994: 406).

Desde el punto de vista de la historia lingüística ${ }^{2}, 1282$ representa un cambio significativo en la vida siciliana. Además de una dirección política diferente, y por lo tanto lingüística (hacia la Península Ibérica más que hacia Francia), que la isla empezó a experimentar y que probablemente permanecería durante algunos siglos, la consecuencia más significativa de las Vísperas ${ }^{3}$, por lo que nos interesa, fue la ruptura de relaciones con la parte meridional de la península. La guerra que por largos decenios enemistó a aragoneses y angevinos, antes y después de la paz de Caltabellotta ${ }^{4}$, transformó en extraño y permanente antagonismo el sentimiento de distinción entre isleños y continentales que ya se habia formado en época nor-

Con el fin de facilitar la comprensión exacta del epígrafe, cuyo interés radica en caracterizar el contexto histórico y lingüistico al que aludiré a lo largo del trabajo, presento a continuación la traducción castellana. ["Asi, como para otras realidades regionales, -escribe Mattesini (1994: 406)-el policentrismo constituye el aspecto principal de la historia politica y cultural de Sicilia -que es todavía, más que en otros lugares, historia tumultuosa de invasiones y conquistas por parte de poblaciones bastante diferentes en cuanto a costumbres, religiones y lenguas-, el carácter isleño del territorio físico, contrariamente a lo que se ha dicho siempre y que todavia se sigue sosteniendo y pensando, no es un rasgo relevante de su cultura, que mas bien muestra una precisa y acentuada identidad autónoma en constante tensión con el resto del territorio nacional.

De hecho, también en cuanto a la tolerancia lingüistica, -sigo citando a Mattesini (1994: 406) - este supuesto aislamiento no encuentra ninguna relación en la realidad, faltándole todo tipo de fundamento cientifico de limites dialectales convencionalmente representado por el Estrecho de Messina (y por las fronteras de la isla), considerando la notable congruencia fenomenológica entre el habla de las dos orillas (y de las islas menores)" ].

- Sobre la historia lingüística y cultural de la isla consúltese con particular atención Vàrvaro (1981), (1984) y (1988: 716-731). Véase también Bruni (1980: 179-278) y Alfieri (1992: 798-860). Para una clasificación de los dialectos sicilianos cfr. Piccitto (1950: 5-34), Pellegrini (1977: 31-32), Ruffino (1984: 161 224). Holtus (1989: XXII y LIII-LVI).

"Se refiere a la llamada "Guerra de las Visperas", que estalló en el mes de abril de 1282, entre angevinos y aragoneses, y duró noventa años. Las dos primeras décadas incidieron considerablemente en la economia y la sociedad de las regiones (Sicilia, Calabria y Campania), que constituyeron el principal escenario de batalla. La guerra se caracterizó por el ensañamiento con que fue conducida por los dos adversarios en combate, y por el carácter de guerrilla que le imprimieron las tropas mercenarias empleadas por los aragoneses (los almogávares).

Para una revisión histórica más detallada del periodo tratado y del resto de la historia siciliana en la edad aragonesa y castellana consúltese Del Treppo (1972), D'Agostino (1979), Del Treppo (1984: 89201), Vitolo (1984: 11-86), Bresc (1986), D'Alessandro (1986: 523-553), Tramontana (1989), Corrao (1991), Galasso (1992) y Vitolo (1994).

4 El tratado de Caltabellotta (en territorio de Agrigento) se firmó en 1302 bajo la presión diplomática del pontifice Bonifacio VIII. Por este acuerdo Federico III de Aragón fue reconocido "rey de Trinacria" con la condicion de que después de su muerte la isla volviera a ser posesión de los angevinos. Pero los hechos fueron muy diferentes ya que después de la desaparición de Federico III. Sicilia mantuvo su rango de ramo de segundón de la dinastía aragonesa. Roberto d'Anjou y luego su nieta Juana movieron ejércitos enteros con la intención de reconquistar la isla; pero todo fue inútil, hasta que la misma Juana con el tratado de Aviñón del 20 de agosto de 1372 reconoció una situación que ya no era posible modificar. 
manda. La intensa unión también lingüística, que los hechos de la dominación habian establecido entre las provincias del sur y Sicilia, fue sin lugar a dudas debilitada: en este periodo no sabriamos indicar ningún fenómeno que haya pasado desde la lengua de un área hasta la de otra.

Es claro que seria un error exagerar la profundidad de la ruptura, al menos por dos razones. La guerra fue larga, y sin embargo no faltó espacio para todo tipo de contactos comerciales en los periodos de paz o en las zonas no directamente interesadas por las operaciones militares. Por otro lado, las mismas hostilidades introdujeron ejércitos napolitanos en la isla y sicilianos en Calabria, provocando contactos que, por muy amigables que hubieran sido, seguian manteniendo las antiguas relaciones.

De todos modos entre 1282 y finales del siglo xv es, como veremos, cuando la romanización de los enclaves grecófonos del mesinés y del "reggino» logran importantes $y$, a veces, definitivos progresos, aunque en esta última área no fue completa. Por lo tanto no carece de significado el hecho de que el dialecto neolatino que sustituye al griego en ambos lados del Estrecho de Messina sea substancialmente el mismo. Eso es ocasionado sin duda por la fuerte cercanía existente entre los dialectos romances de la extrema Calabria y de la Sicilia nororiental; pero el hecho de que las potenciales divergencias no se hayan acentuado implica que las relaciones entre las poblaciones de las dos riberas no se interrumpieran, sea por los ligámenes familiares o por los comerciales y los patrimoniales: por ejemplo, algunas entidades eclesiásticas poseian bienes en las dos partes. $Y$ es posible que ya existiera la corriente migratoria estacional de campesinos que provenían de Calabria para trabajar en las zonas cerealícolas de la Sicilia interna, corriente que conocemos en los periodos sucesivos y que explica la presencia de términos agrícolas característicamente sicilianos en Calabria. Si pensamos en el caso del arabismo gebbia 'tina grande de albañilería donde se conserva el agua para la irrigación', seguramente no autóctono más allá del Estrecho de Messina, donde sobrevive aún en los alrededores de Catanzaro, en el corazón de la región calabresa, en la forma siciliana y también en los de Mormanno, en los extremos límites septentrionales de la misma región, en la de cibbia, cebbia ${ }^{5}$.

La creación de un reino autónomo tuvo todavía consecuencias lingüísticas que no podemos obviar. En época normanda y sueva la cancillería había usado siempre el latín, desde el primer momento junto al árabe y al griego. El siciliano había conquistado un espacio como lengua escrita sólo en la lírica. Cuanto más se reducia el espacio del árabe y del griego, más puesto ocupaba el latín. La situación cambia en la época aragonesa. Durante estos dos siglos nadie usurpa al latín su posición en las funciones escritas de mayor prestigio: la legislación real permanece por ejemplo en latín y en latín es la producción cultural más destacada, desde

${ }^{5}$ Cfr. Rohlis (1977a: 173). 
los escritos humanísticos y teológicos hasta los de derecho poco comunes. A pesar de la eliminación o reducción de las lenguas árabe y griega, el latín no permanece como única lengua escrita, porque también el siciliano se afirma en dominios y con funciones bien lejanas de la lírica áulica o de la transcripción-elaboración de productos populares.

En los últimos decenios del siglo xill el siciliano aparece usado sólo en la creación lírica, aunque sea con un prestigio que va más allá del Estrecho de Messina, al menos según las circunstancias que en esta lengua han redactado algunas de las composiciones transcritas entre 1279 y 1300 en los memoriales de los notarios boloñeses ${ }^{6}$, que en el ámbito del siglo XIII encontramos rimas umbras con sicilianismos $^{7}$. Y el Teniteve messere, septentrional pero con sicilianismos ${ }^{8}$; de 1300 existe un texto aretinizado de la siciliana Molto à ch'io non cantai ${ }^{9}$, y todavía más tarde, entre 1310 y 1330 , en el veneciano Zibaldone da Canal se encuentra la Dio d'amore, en veneciano pero con sicilianismos ${ }^{10}$, y el soneto Tenpo vien che sallie $e$ che desende, que Avalle considera siciliano traducido al veneto ${ }^{11}$. Todavía en 1301 aproximadamente Stefano Protonotaro componía Pir meu cori alligrari ${ }^{12}$, última réplica de la escuela poética siciliana, que - a lo mejor - es la que nos ha llegado en más puro aspecto dialectal. Pero estos son los ecos finales de un prestigio que el siciliano había ganado en sede lírica durante la edad sueva y que ahora se puede considerar un fenómeno del pasado, determinado por las palabras de Dante del De vulgari eloquentia:

"Nam videtur sicilianum vulgare sibi famam pre aliis asciscere, eo quod quicquid poetantur Ytali sicilianum vocatur, et eo quod perplures doctores indigenas invenimus graviter cecinisse» 13 .

Pero Dante distinguia bien entre lengua lírica y hablada:

"Et dicimus quod, si vulgare sicilianum accipere volumus, secundum quod prodit a terrigenis mediocribus, ex ore quorum iudicium elicendum videtur, prelationis honore minime dignum est, quia non sine quodam tempore profertur, ut puta ibi:

Tragemi d'este focora se t'este a boluntate.

Cfr. Caboni (1941).

Editadas por I. Baldelli, en "Studi di filologia italiana" XXIV (1966: 5-10); ahora en Baldelli (1971: 255 y ss.)

* Cfr. Le edizioni a stampa del testi ad uso del TLIO, Firenze, 1977, n. 1635.

9 Ib. n. 1147.

10 Cfr. ed. A. Stussi (1957: 112-117).

1 Cfr. ed. G. Contini (1960: 130-133); la fecha es la de Le edizioni cit., n. 1612, donde ha sido dada con un punto interrogativo.

12 Ib., p. 112.

13 El texto [1. xii.] es el editado por A. Marigo, Firenze, 1968. ["Considerando que el vulgar de Sicilia se atribuye fama por encima de los demás, por el hecho de que todo lo que los italianos componen poéticamente se llama siciliano, y por el hecho de que muchísimos maestros, de ese país nativo, encontramos haber cantado con grandiosidad»] 
Si autem ipsum accipere volumus, secundum quod ab ore primorum Siculorum emanat, ut in preallegatis cantionibus perpendi potest, nichil differt ab illo quod laudabilissimum est $"{ }^{14}$.

Más que detenernos en la evidencia de diferencias diastrásticas entre el siciliano de los primi y el de los mediocres, que tal vez no es más que - según Dante - la distinción entre dos niveles estilísticos de la lengua poética. El párrafo debe ser leido como el epicedio de una función culta que el siciliano había ganado dentro de los limites restringidos y prestigiosos de la lírica en la corte sueva y que no volvería más.

Alrededor del mismo periodo el siciliano empieza, aparentemente desde el inicio, su camino hacia las funciones de la lengua escrita, con un cierre total en los límites de la isla, bastante diferente de lo que habia pasado antes, pero con la adquisición de dominios mucho más amplios y de mayor duración.

El arranque de esta nueva trayectoria es más modesto. El texto en prosa más antiguo que conocemos es, por lo que parece, el de dos recetas rústicas, una de las cuales enseña las prescripciones mágicas para casarse, la otra, cómo se prepara el color turquesa. Escribas ignotos las han catalogado, junto con una receta médica en latín bastante rústico, sobre el folio de guarda de un lapidario francés ${ }^{15}$. Los dos textos son breves y de interés exclusivamente lingüístico, pero prueban que era posible usar el siciliano escrito en un dominio práctico y muestran que se formaba rápidamente una tradición gráfica, a su vez testigo de una difusión cada vez más amplia del uso escrito del siciliano ${ }^{16}$. Pagliaro ha mostrado que el texto más antiguo no señala casi nunca las consonantes dobles, usa $b$ como inicial en bisca, binu y después $r$ en erba, escribe $n$ por [ñ], c o $g$ per [č]; por el contrario el texto más tardío tiene las consonantes dobles, usa $v$ en lava, vassellu, vidi, escribe nni por [ñ], ch por [č]. Descuidando por el momento la nueva literatura en siciliano, cuyos incunables Dialogu de sanctu Gregoriu ${ }^{17}$, Istoria di Eneas ${ }^{18}$ y Libru di

\footnotetext{
${ }^{14}$ [ Y $Y$ afirmo que si se quiere tomar el vulgar siciliano en el sentido de lo que proviene de los regionales de mediana condición, del habla de los cuales evidentemente se tiene que extraer un juicio, eso no es por lo tanto digno del honor de preferencia, desde el momento que no se profiere sin un cierto temor. como por ejemplo en el verso:

Tragemi d'este focora, se t'este a boluntate.

Pero si queremos tomarlo en el sentido de lo que fluye de la boca de los más respetables sicilianos. como se puede bien observar en las suscitadas canciones, en nada difiere del que es el mas digno de elogio (laude)"].

15. Hoy en Paris, Bibl. Nacional, ms. lat. 14470; ed. por A. Pagliaro (1956: 187-198).

16. Los textos sicilianos en alfabeto griego son relativamente numerosos: la lista más amplia (que incluye textos calabreses) puede verse en Caracausi (1975: 107 108), al cual se puede sumar Parlangèli (1962: 462-468), con las indicaciones dadas en la cita. Pagliaro (1953: 284-285) ha individuado como causa del uso del alfabeto griego: "una di queste due circostanze: poca dimestichezza con il latıno [...] oppure esigenza di uniformita grafica, quando il rimanente del codice era in lingua e caratteri greci»: [ "una de estas dos circunstancias: poca confianza en el latín [...] o al contrario exigencia de uniformidad gráfica, cuando el resto del código era en lengua y caracteres griegos"].

17 Cfr. Santangelo (1933).

18 Cfr. Folena (1956)
} 
Valiriu Maximu ${ }^{19}$ pertenecen a los primeros cuatro decenios del siglo XIV ${ }^{20}$, nos interesa aquí destacar que 1320 marca la aparición de un amplio texto prosístico en siciliano, los Capitula edita nuper per curiam super cassia ('caja, gabela') dicta propter guerram imponenda in tota Sicilia ${ }^{21}$. Este texto es el primero que conocemos en el que "la Curti di lu signuri Re» - como se dice - publicara disposiciones fiscales en la lengua cotidiana, instaurando una costumbre que encuentra confirmación en 1328 en Girgenti ${ }^{22}$, en 1332 en Palermo ${ }^{23}$, en 1338 aproximadamente en la pandecta de las carnicerías de Messina ${ }^{24}$, en 1341 en los capítulos suntuarios de Palermo ${ }^{25}$, en 1345 en los capítulos de la gabela del zágatu ('mercería, 'salchichería') emitidos por la corte real en Catania ${ }^{26}$, en 1349 y 1351 en los capitulos frumentarios de Palermo ${ }^{27}$. No seguiremos más allá la difusión del uso, pero es evidente que las disposiciones prácticas, y en particular las fiscales, emitidas por las cancillerias tanto reales como ciudadanas, están escritas en lengua siciliana, que recibe plena validez como lengua de uso administrativo. Todavia mejor se entiende cómo el siciliano haya sido usado en las costumbres de las distintas ciudades del reino, sólo que en este caso no es fácil fechar la primera extensión escrita de tales textos, que nos han llegado en general en reelaboraciones más tardías. La afirmación del siciliano en estos dominios hace fácilmente comprensible que el mismo resulte adecuado en la actuación jurídica privada más solemne, la disposición testamentaria: ya en 1349 el sacerdote Guglielmino de Banbacara testa en Catania en vulgar, aún siendo clérigo y por cuanto lo hace en el interés de un monasterio como San Nicolò la Arena, ni Filippo de Santa Sofía, publicus civitatis Cathanie notarius, encuentra nada que decir ${ }^{28}$. No podemos más que asombrarnos si en 1361 encontramos una relación de la valoración de una casa en Palermo ${ }^{29}$ y todavía menos si en 1365 se verbaliza en siciliano el testimonio de Perronus de Raya de Alcamo ${ }^{30}$.

Se entiende que el siciliano debería aparecer también, admisiblemente, en la correspondencia: no asombra que el 26 de marzo de 1338 Giovanni de Chiaramonte, siniscalco del reino de Sicilia, use el siciliano para escribir desde Agrigento a Rainuccio de Gimilia, su procurador ${ }^{31}$, y que desde aquel momento las cartas de

${ }^{9}$ Cfr. Ugolini (1967) y Mattesini (1991).

${ }^{20}$ Para estas obras reenvio al estudio de Bruni (1980). También sobre sus fechas la contribución de Bruni es fundamental.

21 Cfr. "Volgare nostro siculo" [=VNS] Crestomatia de textos sicilianos del siglo xIV. Parte I, ed. Li GotTI (1951: 24-32).

2. Cfr. "Bollettino del Centro di Studi Filologici e Linguistici Siciliani» [=CSFLS] 1 (1953: 239-240)

Cfr. "Bollettino CSFLS»10 (1969: 413).

${ }^{4}$ Cfr. VNS, pp. 55-63.

Cfr. VNS, pp. 34-35

Cfr. VNS, pp. 37-40

Cfr. VNS, pp. $42-44$ y $68-70$

${ }^{28}$ Cfr. VNS, pp. 48-50; otro testamento de 1371 es editado por $P$. Palumbo en "Bollettino CSFLS" 5 , (1957: 357).

29 Editado por P. Palumbo en «Bollettino CSFLS"1 (1953: 240-241).

36. Editado por C. Trasselli en "Bollettino CSFLS» 3 (1955: 317)

${ }^{31}$ Cfr. VNS, pp. 84-85. 
carácter oficial aumenten considerablemente - Li Gotti las llama "cartas ordenaciones"-, incluida una del rey Federico IV en 1373, más tarde ${ }^{32}$; del resto la más antigua carta privada que conocemos, del 15 de septiembre de 1341, es la enviada al rey Pedro II desde Santa Lucia del Mela de Raimondo Peralta ${ }^{33}$. Que estos primeros textos relacionen inmediatamente la curia real y los rangos más altos consigue con mayor facilidad que los documentos de tales niveles fueran conservados hasta hoy, prefiriendo los de origen y destinación más humildes, pero tiene todavía la ventaja, para nosotros, de señalarnos la difusión del uso de la lengua siciliana para el soberano, los nobles de cada nivel, las administraciones ciudadanas y el alto y bajo clero, en suma para todos los que escriben. Para el clero tenemos el significativo ejemplo del abate Angelo Senisio, que ya en 1348 se muestra atento al léxico vulgar en su diccionario latino titulado Liber declarus y que en 1370 recibe una carta en siciliano enviada desde Gaeta por fray Benedetto de Bulgarino ${ }^{34}$, que es sólo el primer texto de un breve epistolario recogido por Li Gotti, que muestra que para el docto abate de San Martino delle Scale era normal escribir en siciliano; además en siciliano escribía más de una vez, entre 1370 y 1379, fray Stefano al prior de Montecassino ${ }^{35}$. Entre 1372 y 1381 el abate Senisio tenía en siciliano el propio libro de cuentas ${ }^{36}$, demostrando con ese mismo que esta lengua podía ser utilizada en una vasta gama de funciones, de las más prácticas y privadas a las privadas pero formales.

Un monasterio benedictino como San Martino delle Scale reservaba todavía al latín funciones litúrgicas, exhortativas y disciplinares, que en otro sitio podian en parte ser practicadas en vulgar. En 1343 los capítulos de la primera compañia de disciplinantes de Palermo, organización que nacía de una espiritualidad más moderna y popular, vienen redactados en siciliano ${ }^{37}$ y aparecen en la misma lengua, tal vez en 1344, las constituciones del abate y de los monjes de Santa María de Licodia $^{38}$, lo que es extremamente interesante, porque Santa María y San Nicolò la Arena estaban en una estrecha relación que se iba resolviendo a favor del segundo, y eran los mayores centros benedictinos de Sicilia, mostrándose asi todavia más abiertos al siciliano que a la filiación palermitana.

La apertura de los ambientes religiosos de la isla a la cultura en vulgar desde muchos decenios antes de estos textos resulta más bien del estudio de Francesco Bruni (1980). De estas obras no parece necesario hablar de nuevo aquí, si no para señalar que antes o contemporáneamente a la introducción del siciliano en los dominios escritos citados, ese iba asumiendo las funciones de prosa literaria en tres traducciones: los ya citados Santu Gregoriu, Eneas e Valiriu Maximu. En

32 Cfr. VNS, pp. 94-95; se adjuntan las cartas de la reina de Aragón Eleonora, editadas por Giunta (1973: 346-353); más antigua es la carta de la misma Eleonora editada por Romano (1973: 387-391).

33 Cfr. VNS. pp. 114-115.

34 Cfr. VNS, pp. 126-133.

Cfr. VNS, pp. 151 y $158-163$.

36 Cfr. A. Giuffrida, en "Bollettino CSFLS" 12 (1973: 151-166).

37 Ctr. Branciforti (1953: 3-26).

38 lb. pp. 27-34. 
1358 seguía la Conquesta di Sichilia, es decir la versión de la crónica de Goffredo Malaterra, en 1368 la versión de la Mascalcia de Giordano Ruffo, y finalmente en 1373 se tenía una amplia obra, esta vez en original, la Sposizione del Vangelo della Passione secondo Matteo ${ }^{39}$. No recordaré los otros textos, en general también traducidos, pero es significativo que se trate por lo tanto de prosa y que el siciliano se vea ya casi imposibilitado al uso poético: sólo en 1354 encontramos el llamado Lamento di parte siciliana ${ }^{40}$ y del siglo XIV queda solamente el Pianto di Maria y el soneto Sta pistula mandata mustra comu; tenemos que esperar al siglo XV para una producción más abundante, que corresponda con una traducción más radical, que confirme - además - la ruptura, también lingüística, con la tradición sueva ${ }^{41}$.

En conclusión, a la llegada a Sicilia del duque de Montblanc al final del siglo XIV, el siciliano es prácticamente lengua escrita oficial del reino junto al latín, con una vastísima gama de textos administrativos, culturales y privados. El latín sigue siendo la lengua de la liturgia, de la cultura religiosa y de la laica de nivel académico, de las expresiones más solemnes y grandielocuentes de la legislación y del derecho, pero no es más, desde hace tiempo la única lengua escrita y va reduciendo notablemente sus usos. El examen de una serie compacta de cartas enviadas por el duque de Montblanc en el verano de $1394^{42}$ lleva a la conclusión de que la tendencia dominante en la cancillería ducal era la de usar el latín para los documentos estrictamente laborales y de derecho público, como las investiduras feudales, mientras se recurría al siciliano para los documentos referidos a privados y terceras personas, además del remitente y destinatario. Entre las lenguas escritas, el latín se reserva así mismo los usos más "altos", pero se trata de ámbitos siempre más restringidos.

\section{BIBLIOGRAFIA}

ALFIERI, G. (1992): La Sicilia, en Bruni (1992:798-860).

AMARI, M. (ed.) (1937): Storia dei musulmani di Sicilia, Catania

AmbrosinI, R. (1977): Stratigrafia lessicale di testi siciliani dei secoli XIv e XV. Palermo.

BALDELLL, I. (1971): Medioevo volgare da Montecassino all'Umbria, Bari.

BartolotTA, S. (1995): I/ dialetto di Castrofilippo, Catania.

BARtolotTA, S. (1999): Il linguaggio degli studenti di Agrigento, en Caratu (1999: 165-180).

Bartolotta, S. (2000): Pertil de historia lingüistica de Sicilia en la edad aragonesa y castellana, memoria de investigación, UNED, Madrid.

Bartolotta, S (2003): La versión española de "Candido" de Leonardo Sciascia: estudio lingüístico de los regionalismos, tesis doctoral, UNED, Madrid.

BARTOLOTIA, S. (2003): Lengua original y traducción castellana en "Candido" de Leonardo Sciascia, en “Epos», XIX, pp. 247-258.

34 Cfr. Palumbo (1954-1957).

40 Cfr. Cusimano (1951: 23-30); esta obra falta en Le edizioni a stampa cit. Para los textos antes mencionados reenvio al estudio cit. de Bruni (1980)

4 Conviene recordar que en la Sicilia del siglo xiv se ha producido todavia poesía en lengua provenzal: cfr. Ruggieri (1953: 204-232)

42 Cfr. Vàrvaro $(1977: 1-7)$ 
Bartolotta, S. Lunetta, S. Messina, C. Tedeschi, P. Virgillitto, S. (1993): "Confessionale /"”: Analisi linguistica e confronto con "Libru di lu transitu et vita di messer Sanctu Iheronium" ed "Ordini di la confessioni Renovamini», Catania.

BatTAGLIA, S. (1962-2002): Grande dizionario della lingua italiana, 21 vols., Torino.

BeccaRIA, G. L. (1968): Spagnolo e spagnoli in Italia, Torino.

Beretta Spampinato, M. (1980): La prosa del '500, en "Storia della Sicilia" IV, Palermo.

BrANCIFORTI, F. (ed.) (1953): Regole, costituzioni. confessionali e rituali, en "Collezione di testi siciliani dei secoli $x i v$ e $x v>3$, Palermo.

BRESC. H. (1971): Livre et société en Sicile (1299-1499), Palermo

BREsC. H. (1986): Une monde méditerranéen. Economie et société en Sicile. 1300-1450, Roma-Palermo.

Bresc. H. Goltein. S. D. (1970): Un inventaire dotal des juifs siciliens (1479), en "Mélanges d'archéologie et d'histore de l'Ecole française de Rome" 82 .

BRincat, G. (1977): Malta e Pantelleria: alla ricerca di un sostrato comune, en "Journal of Maltese Studies" 11.

Bruni, F. (1980): La cultura e la prosa volgare nel '300 e nel '400, en "Storia della Sicilia» IV. Palermo.

BRuni, F. (ed.) (1973): Libru di li vitii et di li virtuti, en "Collezione di testi siciliani dei secoli XIV e XV", 3, Palermo.

BRUNI, F. (1992): L'italiano nelle regioni. Lingua nazionale e identità regionali, Torino.

CABONI, A. (1941): Antiche rime italiane tratte dai Memoriali bolognesi, Modena.

Caracausi, G. (1975): Byzantino-Sicula II, Palermo

Caratu, P. (ed.) (1999): Linguistica Italiana Meridionale IV-V 1996/1997, Bari.

Catalano, M. (1950): La leggenda della Beata Eustochia da Messina, Messina-Firenze.

Contini, G. (ed.) (1960): Poeti del Duecento, I, Milano-Napoli.

CORRAO, P. (1991): Governare un regno, Napoli.

Cusimano, G. (ed.) (1951): Poesie siciliane dei secoli XIV e XV, en "Collezione di testi siciliani dei secoli XIV e XV» 3, Palermo.

D Agostino, G. (1979): Parlamento e società nel regno di Napoli. Secoli xv-Xvi, Napoli.

D Alessandro, V. (1963): Politica e societa nella Sicilia aragonese, Palermo.

D AlEssAnDRO, V'. (1986): I/ Mezzogiomo dagli Angioini agli Aragonesi en «La storia. I grandi problemi dal Medioevo allEtà contemporanea", vol. II, Torino.

Del Treppo, M. (1972): I mercanti catalani e l'espansione della corona aragonese nel secolo xV. Napoli.

DEL TREPPO, M. (1984): I/ regno aragonese en Galasso-Romeo 1984.

Di Marzo, G. (1869-1886): Diari della città di Palermo dal secolo XVi al xix. Palermo.

DRAE (1998): Diccionario de la lengua de la Real Academia Española, 2 vols., XXI ed., Madrid.

FAvarA, A. (1957): Corpus di musiche popolari siciliane, Milano.

Folena, G. (ed.) (1956): La istoria di Eneas vulgarizata per Angilu di Capua, en "Collezione di testi siciliani dei secoli XIV e XV»7, Palermo.

Galasso, G. (1992): I Regno di Napoli. Il Mezzogiorno angioino e aragonese (1266-1494), Torino.

Galasso, G. Romeo, R. (ed.) (1984): Storia del Mezzogiorno, vol. IV. Roma-Napoli.

GIUNTA, F. (1973): Un gruppo di lettere in siciliano di Eleonora d'Aragona (1375), en "Bollettino CSFLS" 12, Palermo.

GotteIN, S. D. (1967): A Mediterranean Society. The Jewish Communities of the Arab World as portrayed in the Documents of the Cairo Geniza. Berkeley-Los Angeles-London.

Guillou, A. (1963): Les actes grecs de S. Maria Di Messina, Palermo.

Gultıou, A. (1976): Aspetti della civiltà bizantina in Italia, Bari.

Holtus, G. (1989): Per una carta dialettologica italiana, en Holtus-Metzeltin-Pfister (1989: XXXII Y LIIILVI).

Holtus, G. Metzel.tin, M. Pfister, M. (ed.) (1989): La dialettologia oggi. Studi offerti a Manlio Cortelazzo, Tübingen.

KELLNER, H. (1972): Die albanische Minderheit in Sizilien, Wiesbaden.

LA MANTIA, G (1907): I capitoli delle colonie greco-albanesi di Sicilia nei secoli xv e XVI, Palermo.

Lagumina, B. Lagumina, C. (1884-1895): Codice diplomatico dei Giudei di Sicilia, Palermo.

LEONE, A. (ed.) (1990): // vocabolario siciliano-latino di L. C. Scobar, Palermo.

LI GotTi, E. (ed.) (1951): Volgare nostro siculo [= VNS] I, Firenze.

MANGION, G. (1973): L'italiano a Malta, en "Storia linguistica dell'Italia del Novecento", Roma.

MANGion. G. (1974): Appunti di storia linguistica maitese, en "Dal dialetto alla lingua", Pisa.

Mattesini, E. (ed.) (1991): Valeriu Maximu translatatu in vulgar messinisi per Accursu di Cremona, Pa. lermo.

Mattesini, E. (1994) Sicilia, en Serianni-Tritone (1994: Vol. III: 406-32). 
Migliorinı, B. (1978): Storia della lingua italiana, 2 vols., Firenze (reim. con la introducción de G. Ghinassi).

NALL, P. (1939): Saggio bibliografico sui testi a stampa in dialetto siciliano, en «Scritti vari dedicati a Mario Armanni», Milano.

MOCCIARO, A. G. (1975): Vocabolari siciliani manoscritti inediti, en "La ricerca dialettale" 1.

PAGliARO, A (ed.) (1953): Saggi di critica semantica, Messina-Firenze.

PAGLIARO, A. (1956): Nuovi saggi di critica semantica, Messina-Firenze.

Palumbo, P. (ed.) (1954-1957) Sposizione del Vangelo della Passione secondo Matteo, Palermo.

PARLANGELI, O. (1962): Testi siciliani in caratteri greci, en "Bollettino CSFLS"7, Palermo.

PELlegRINI, G. B. (1972): Gli arabismi nelle lingue neolatine. Brescia.

Pellegrini, G. B. (1977): Carta dei dialetto d'italia, Pisa.

Petrotta. S. (1966): Albanesi di Sicilia, Palermo.

PIccitto, G. (1950): La classificazione delle parlate siciliane e la metafonesi in Sicilia, en "Archivio storico per la Sicilia orientale", serie IV, III.

PITRE, G. (1928): Supplemento ai dizionari siciliani, en "Studi Glottologici Italiani» VIII.

Quattordo Moreschini, A. (ed.) (1984): Tre millenni di storia linguistica della Sicilia (Atti del Convegno delia Società Italiana di Glottologia, Palermo 25-27 marzo 1983), Pisa.

ROHLFS, G. (1962): Nuovi contributi al grecismo della Sicilia nord-orientale, en «Bollettino CSFLS» 8, Palermo.

ROHLFS, G. (1974): Scavi linguistici nella Magna Grecia, Galatina.

RoHlfs, G. (1975): Historische Sprachschichten in modernen Sizilien, München (tr. it. La Sicilia nei secoli, Palermo, 1984).

ROHLFs, G. (1977a): Nuovo dizionario dialettale della Calabria, Ravenna.

RoHLfs, G. (1977b): Supplemento ai vocabolari siciliani, München.

Romano, D. (1973): Un testo in volgare siciliano del 1351, en "Culture regionali e letteratura nazionale. Atti del VII Congresso dell'Associazione Internazionale per gli Studi di Lingua e Letteratura Italiana", Bari.

Rossi, V. (1930): Caio Caloria Ponzio e la poesia volgare letteraria di Sicilia del secolo xv, en sus Scritti di critica letteraria, II, Firenze.

RufFINO, G. (1984): Isoglosse siciliane, en Quattordio Moreschini (1984: 161-224).

RugGieRl, R. M. (1953): La poesia provenzale alla corte di Federico Ill di Sicilia, en "Bollettino CSFLS" 1 , Palermo.

Santangelo, G. (1975): Letteratura in Sicilia da Federico /l a Pirandello, Palermo.

SANTANGElo, S. (ed.) (1933): Libru di lu dialagu de Sanctu Gregoriu traslatatu pir trati lohanni Campulu de Missina, Palermo.

SCADUto, M. (1947): II monachesimo basiliano nella Sicilia medievale, Roma.

SERIANNI, L. Trifone, P. (ed.) (1994): Storia della lingua italiana, 3 vols., Torino.

SORRENTO, L. (1921): La diffusione della lingua italiana nel Cinquecento in Sicilia, Firenze.

StaRRABBA, R. (1873a): Di un documento riguardante la Giudecca di Palermo, en "Archivio Storico Siciliano" 1.

Starrabba, R. (1873b): Processo di fellonia contro frate Simone Del Pozzo vescovo di Catania (1392), en "Archivio Storico Siciliano" I.

StaRrabba, R. (ed.) (1887-1888): Lettere e documenti relativi a un periodo del vicariato della Regina Bianca in Sicilia (1411-1412). Palermo.

Stussi, A. (ed.) (1957): Zibaldone da canal, Venezia.

Tiвy, O. (1954): Il problema della "siciliana" dal Trecento al Settecento, en "Bollettino CSFLS" 2, Palermo.

Traina, A. (1868): Nuovo vocabolario siciliano-italiano, Palermo.

TrainA, A. (1877): Vocabolarietto delle voci siciliane dissimili dalle italiane, Palermo.

TRAMONTANA, S. (1989): Gli anni del Vespro, Bari.

Trassell., C. (1951): Sicilia, Levante e Tunisi nei secoli XIV e XV, Trapani.

Tropea, G. (1975): Per una monografia sul dialetto dellisola di Pantelleria, en «La ricerca dialettale" I.

UGOLINI, F. A. (ed.) (1967): Valeriu Maximu translatatu in vulgar messinisi per Accursu di Cremona, Palermo.

Varvato, A. (1974): Prima ricognizione dei catalanismi nel dialetto siciliano, en "Medioevo romanzo"I VARVARO, A. (1977): Note per la storia degli usi linguistici in Sicilia, en "Lingua Nostra" XXXVIII, Palermo. VARVARO, A. (1981): Lingua e storia in Sicilia. Dalle guerre puniche alla conquista normanna, Palermo. VARVARo, A. (1984): La parola nel tempo. Lingua, società e storia, Bologna.

VArvaro, A. (1988): Aree linguistiche, XII. Sicilia, en "Lexikon der Romanischen Linguistik" 4, Tübingen. Veneziano, A. (1967): Ottave, ed. A. Rigoli, Torino. 
Vitolo, G. (1984): II regno angioino, en Galasso-Romeo 1984.

Vitolo, G. (1994): Corso di Storia I. Medioevo, Milano.

VNS (1951): Volgare nostro siculo, Crestomatía de textos sicilianos del siglo xIV, parte l, ed. E. Li Gotti, Firenze.

VS (1977-2002): Vocabolario siciliano, Vol. I (A-E) ed. G. Piccitto, Catania-Palermo 1977; Vol. II (F-M) ed. G. Tropea, Catania-Palermo 1985; Vol. III (N-Q) ed. G. Tropea, Catania-Palermo 1990; Vol, IV (R-S) ed. G. Tropea, Catania-Patermo 1997; vol. V (Si-Z) ed. S. C. Trovato, Catania-Palermo 2002. 